\title{
Dynamical Properties of Scaled Atomic Wehrl Entropy of Multiphoton JCM in the Presence of Atomic Damping
}

\author{
S. Abdel-Khalek, ${ }^{1,2}$ M. S. Almalki, ${ }^{1}$ and E. Edfawy ${ }^{1,3}$ \\ ${ }^{1}$ Mathematics Department, Faculty of Science, Taif University, Taif, Saudi Arabia \\ ${ }^{2}$ The Abdus Salam International Center for Theoretical Physics, Strada Costiera11, Miramare, Trieste, Italy \\ ${ }^{3}$ Mathematics Department, Faculty of Science, Assuit University, Assuit, Egypt
}

Correspondence should be addressed to S. Abdel-Khalek; sayedquantum@yahoo.co.uk

Received 23 March 2013; Revised 24 June 2013; Accepted 8 September 2013

Academic Editor: Ashok Chatterjee

Copyright (C) 2013 S. Abdel-Khalek et al. This is an open access article distributed under the Creative Commons Attribution License, which permits unrestricted use, distribution, and reproduction in any medium, provided the original work is properly cited.

\begin{abstract}
We study the dynamics of the atomic inversion, scaled atomic Wehrl entropy, and marginal atomic Q-function for a single twolevel atom interacting with a one-mode cavity field taking in the presence of atomic damping. We obtain the exact solution of the master equation in the interaction picture using specific initial conditions. We examine the effects of atomic damping parameter and number of multiphoton transition on the scaled atomic Wehrl entropy, atomic Q-function, and their marginal distribution. We observe an interesting monotonic relation between the different physical quantities in the case of different values of the number of photon transition during the time evolution.
\end{abstract}

\section{Introduction}

Entanglement is a property of correlations between two or more quantum systems [1]. These correlations defy classical description and are associated with intrinsically quantum phenomena. This nonlocal nature of entanglement has also been identified as an essential resource for many novel tasks such as quantum computation, quantum teleportation [2], superdense coding [3], quantum cryptography [4, 5], and more recently, one-way quantum computation [6], and quantum metrology [7]. These quantum information tasks cannot be carried out by classical resources and they rely on entangled states. This recognition led to an intensive search for mathematical tools that would enable a proper quantification of this resource [8]. In particular, it is of primary importance to test whether a given quantum state is separable or entangled.

It is well known that the Jaynes-Cummings model (JCM) becomes more a realistic and experimental model under the effect of damping [9]. Besides the experimental drive, also there exists a theoretical motivation to include relevant damping mechanism to the JC model because its dynamics becomes more interesting. In this regard, many authors have treated the JCM with dissipation by the use of analytic approximations $[10,11]$ and numerical calculations [12-14]. The solution in the presence of dissipation is not only of theoretical interest but also important from a practical point of view since dissipation would be always present in any experimental realization of the model. However, the dissipation treated in the above studies is modeled by coupling to an external reservoir including energy dissipation. As is well known, in a dissipative quantum system, the system loses energy by creating a bath quantum.

Over the last two decades, much attention has been focused on information entropies as a measure of the entanglement in quantum information [1]. In this regard, the von Neumann entropy (NE) [15], linear entropy (LE), and Shannon information entropy (SE) [16] have been frequently used in entanglement discussions concerning a variety of quantum systems. It is worth mentioning that the SE involves only the diagonal elements of the density matrix and in some cases gives information similar to that obtained from the NE. On the other hand, there is an additional entropic quantity, namely, the semiclassical, phase-space atomic Wehrl entropy (AWE) [17-19]. This measure has been successfully applied in 
measuring the entanglement in the JCM when the modes are initially prepared in a finite dimensional trio-coherent state (FTCS) [20]. Also, the dynamical properties of the AWE for a single two-level trapped ion interacting with a laser field has been investigated [21]. It is shown that the AWE gives quantitative (qualitative) information on the entanglement of the bipartite system. Also, the correlation between Wehrl entropy and Fisher information has been investigated based on the Husimi Q-function $[22,23]$ and the atomic $Q$-function [24].

In this article, we consider another extension of the problem by considering what is called the scaled atomic Wehrl entropy associated with the reduced atomic density operator as an entanglement quantifier of a tow-level atom interaction with coherent field under the effect of atomic damping. We focus on the effect of the atomic damping and number of photon transition on the evolution of the atomic inversion, scaled atomic Wehrl entropy, and marginal atomic Q-function.

The paper is organized as follows. In Section 2, the Hamiltonian and master equation of a two-level atom in the presence of atomic damping is introduced, followed by a discussion of the method to calculate the scaled atomic Wehrl entropy and marginal atomic Q-function in Section 3. Numerical results of the calculated scaled atomic Wehrl entropy are presented and compared with the marginal atomic Q-function in Section 4. We conclude in Section 5, with a summary and an outlook.

\section{Model Hamiltonian and Master Equation}

The interaction between radiation and matter is a central problem in quantum optics and information. The simplest physical situation can be successfully described by a rather simplified but nontrivial model which is popularly known as the JCM [9], which describes the interaction between a single two-level atomic system and a quantized radiation mode. Despite its simplicity, the JCM have great significance because technological advances have enabled us to experimentally realize this rather idealized model $[25,26]$ and to verify some of the theoretical predictions. Stimulated by the success of the JCM, more and more researchers have paid special attention to extending and generalizing the model in order to explore certain new quantum effects [27]. One possible generalization is the multiphoton JCM:

$$
\begin{aligned}
\widehat{H} & =\widehat{H}_{0}+\widehat{H}_{\mathrm{in}} \\
& =\omega\left(\frac{\widehat{\sigma}_{z}}{2}+\widehat{a}^{\dagger} \widehat{a}\right)+\lambda\left[\widehat{a}^{\dagger^{k}}|g\rangle\left\langle e\left|+\widehat{a}^{k}\right| e\right\rangle\langle g|\right],
\end{aligned}
$$

where the two-level atom of transition frequency $\omega$ interacts resonantly with the field which is described by the bosonic operators $\hat{a}^{\dagger}$ and $\hat{a}$, the creation and annihilation operators. The two-level states are $|g\rangle$ and $|e\rangle, \widehat{\sigma}_{z}=|e\rangle\langle e|-| g\rangle\langle g|$ is the population inversion operator, $k$ is the number of photons transition.
We consider the master equation which describes only the atomic damping:

$$
\begin{aligned}
\frac{d \hat{\rho}(t)}{d t}= & \frac{-i}{\hbar}\left[\widehat{H}_{\mathrm{in}}, \hat{\rho}(t)\right] \\
& +\gamma\left[2 \widehat{\sigma}_{-} \hat{\rho}(t) \widehat{\sigma}_{+}-\widehat{\sigma}_{+} \widehat{\sigma}_{-} \widehat{\rho}(t)-\widehat{\rho}(t) \widehat{\sigma}_{+} \widehat{\sigma}_{-}\right] .
\end{aligned}
$$

The exact solution for this equation in the case of a high-Q cavity $\gamma / \lambda \ll 1$ is obtained by analytic method by using the dressed state representation [28, 29], that is, representation consisting of the complete set of eigenstates of the Hamiltonian (1) is used, which is given by

$$
\left|\beta_{n}^{ \pm}\right\rangle=\frac{1}{\sqrt{2}}(|e, n\rangle \pm|g, n+k\rangle), \quad(n=0,1,2, \ldots)
$$

with

$$
\begin{gathered}
\widehat{H}_{\text {in }}\left|\beta_{n}^{ \pm}\right\rangle= \pm \mu_{n}\left|\beta_{n}^{ \pm}\right\rangle \text {where the eigenvalues are } \\
E_{n}^{ \pm}= \pm \lambda \sqrt{\frac{(n+k) !}{n !}} .
\end{gathered}
$$

To derive (2) in the dressed state representation, we write the atomic operators in terms of the dressed state representation as

$$
\begin{aligned}
& \widehat{\sigma}_{+}= \frac{\left(\left|\beta_{0}^{+}\right\rangle\left\langle g, 0|+| \beta_{0}^{-}\right\rangle\langle g, 0|\right)}{\sqrt{2}} \\
&+\frac{1}{2} \sum_{n=1}^{\infty}\left[\left|\beta_{n}^{+}\right\rangle\left\langle\beta_{n-1}^{+}|-| \beta_{n}^{+}\right\rangle\left\langle\beta_{n-1}^{-}\right|\right. \\
&\left.+\left|\beta_{n}^{-}\right\rangle\left\langle\beta_{n-1}^{+}|-| \beta_{n}^{-}\right\rangle\left\langle\beta_{n-1}^{-}\right|\right], \\
& \widehat{\sigma}_{+} \widehat{\sigma}_{-}=\frac{1}{2} \sum_{n=0}^{\infty}\left[\left|\beta_{n}^{+}\right\rangle\left\langle\beta_{n}^{+}|+| \beta_{n}^{+}\right\rangle\left\langle\beta_{n}^{-}\right|\right. \\
&\left.+\left|\beta_{n}^{-}\right\rangle\left\langle\beta_{n}^{+}|+| \beta_{n}^{-}\right\rangle\left\langle\beta_{n}^{-}\right|\right],
\end{aligned}
$$

and using the following representation:

$$
\frac{d}{d t}(\widehat{x}(t))=e^{i H t} \frac{d \hat{\rho}(t)}{d t} e^{-i H t}+i[H, \widehat{x}(t)],
$$

where $\widehat{x}(t)$ is an arbitrary operator of combined system. We can rewrite the master equation (2) in terms of the dressed state representation. Then, we choose any initial state, here we let the atom be initially in the excited state, that is, $|e\rangle$, while the radiation mode is in a coherent state:

$$
\begin{gathered}
\hat{\rho}^{F}(0)=|\alpha\rangle\left\langle\alpha\left|=\sum_{m, n}^{\infty} b_{n}(\alpha) b_{m}^{*}(\alpha)\right| n\right\rangle\langle m|, \\
b_{n}(\alpha)=\alpha^{n} \sqrt{\frac{e^{-|\alpha|^{2}}}{n !}} .
\end{gathered}
$$


The initial system density matrix $\widehat{\rho}(0)$ can be written in terms of the eigenvector equation (3) as follows:

$$
\begin{aligned}
\hat{\rho}(0)=\frac{1}{2} \sum_{n, m=0}^{\infty} b_{n}(\alpha) b_{m}^{*}(\alpha) & \\
\times & {\left[\left|\beta_{n}^{+}\right\rangle\left\langle\beta_{m}^{+}|+| \beta_{n}^{+}\right\rangle\left\langle\beta_{m}^{-}\right|\right.} \\
& \left.+\left|\beta_{n}^{-}\right\rangle\left\langle\beta_{m}^{+}|+| \beta_{n}^{-}\right\rangle\left\langle\beta_{m}^{-}\right|\right] .
\end{aligned}
$$

The solution of master equation in the high-Q limit with previous initial states is given by

$$
\begin{aligned}
\hat{\rho}(t)=\sum_{n, m=0}^{\infty}\{ & A_{n, m}(t)|n, e\rangle\langle e, m| \\
& +B_{n, m}(t)|n, e\rangle\langle g, m+k| \\
& +B_{n, m}^{*}(t)|n+k, g\rangle\langle e, m| \\
& \left.+D_{n, m}(t)|n+k, g\rangle\langle g, m+k|\right\},
\end{aligned}
$$

where

$$
\begin{gathered}
A_{n, m}(t)=\frac{1}{2} \chi_{n} \delta_{n m} \\
+\left[\frac{1}{2} b_{n}(\alpha) b_{m}^{*}(\alpha) 2 \cos \left(\mu_{n} t\right)\right. \\
\left.\quad \times \cos \left(\mu_{m} t\right)-\delta_{n m}\right] e^{-(\gamma t)}, \\
B_{n, m}(t)=i b_{n}(\alpha) b_{m}^{*}(\alpha)\left[\cos \left(\mu_{n} t\right) \sin \left(\mu_{m} t\right)\right] e^{-(\gamma t),} \\
D_{n, m}(t)=\frac{1}{2} \chi_{n} \delta_{n m} \\
-\left[\frac{1}{2} b_{n}(\alpha) b_{m}^{*}(\alpha) 2 \cos \left(\mu_{n} t\right)\right. \\
\left.\quad \times \cos \left(\mu_{m} t\right)-\delta_{n m}\right] e^{-(\gamma t)},
\end{gathered}
$$

with

$$
\chi_{n}=e^{-(\bar{n}+\gamma t)}\left(\frac{\bar{n}}{\gamma t}\right)^{n / 2} I_{n}(2 \sqrt{\bar{n} \gamma t})
$$

where $I_{n}(\cdot)$ is the modified Bessel function and $\bar{n}=|\alpha|^{2}$.

All information about the system is involved in the total density matrix (9). As we mainly deal with the atomic subsystem, we evaluate the atomic reduced density matrix $\widehat{\rho}_{A}(t)$ via the relation:

$$
\widehat{\rho}_{A}(t)=\operatorname{tr}_{F}\{\hat{\rho}(t)\},
$$

where the subscript $F$ means that the trace is taken over the field.

We close this section by presenting the concept of the atomic population inversion which is considered as the simplest important quantity to be calculated. It is related to the difference between the probabilities of finding the atom in the exited state and ground state:

$$
\widehat{\rho}_{z}(t)=\frac{1}{2}\left[\left\langle e\left|\widehat{\rho}_{A}(t)\right| e\right\rangle-\left\langle g\left|\widehat{\rho}_{A}(t)\right| g\right\rangle\right] .
$$

We use the results obtained in this section to make a comparative study between the atomic inversion, marginal atomic Q-function, and scaled AWE in the following sections.

\section{Atomic Wehrl Entropy and Marginal Distribution}

The atomic Wehrl entropy is a useful tool to investigate dynamical properties of quantum systems that contains all the information about the system dynamics, being completely equivalent to the atomic density operator and it can be interpreted as being an information measure for such joint measurement. The atomic Wehrl entropy can be used as a quantifier of the quantum entanglement between twolevel atom and coherent field. Also, the information about the system dynamics and entanglement can be obtained through the marginal distribution of the atomic $Q$-function and atomic Wehrl entropy

In this section, we investigate the marginal atomic Qfunction and atomic Wehrl entropy AWE. We start our investigation by defining the atomic $Q$-function as [17]

$$
Q_{A}(\Theta, \Phi, t)=\frac{1}{2 \pi}\left\langle\Theta, \Phi\left|\widehat{\rho}_{A}(t)\right| \Theta, \Phi\right\rangle,
$$

where $\hat{\rho}_{A}(t)$ is the density matrix which is given in (12) and $|\Theta, \Phi\rangle$ is the atomic coherent state expressed as

$$
|\Theta, \Phi\rangle=\cos (\Theta / 2)|e\rangle+\sin (\Theta / 2) e^{i \Phi}|g\rangle,
$$

where $0 \leq \Theta \leq \pi, 0 \leq \Phi \leq 2 \pi$. Definition (14) means that two different spin coherent states overlap unless they are directed into two antipodal points on the sphere [17].

The scaled atomic Wehrl entropy can be written in terms of the atomic $Q$-function as $[17,30]$ :

$$
\begin{aligned}
\mathrm{SS}_{\mathrm{AW}}(t)= & \frac{1}{1 /(2 \ln (2))-1} \\
& \times\left\{\ln (2 \pi \sqrt{e})+\int_{0}^{2 \pi} \int_{0}^{\pi} Q_{A}(\Theta, \Phi, t)\right. \\
& \left.\times \ln Q_{A}(\Theta, \Phi, t) \sin \Theta d \Theta d \Phi\right\} .
\end{aligned}
$$

It is worth noting that from the definition (16), the $\mathrm{S}_{\mathrm{AW}}(t)$ cannot be negative as a result of $Q_{A}$ being a nonnegative function. As it is generally difficult to find a closed form for the $\mathrm{S}_{\mathrm{AW}}(t)$, numerical techniques have to be used. Nevertheless, at particular values of the interaction parameters the exact form can be obtained. The shifted (scaled) $\mathrm{S}_{\mathrm{AW}}(t)$ satisfies the following inequality:

$$
0 \leq \mathrm{SS}_{\mathrm{AW}}(t) \leq \ln 2 .
$$




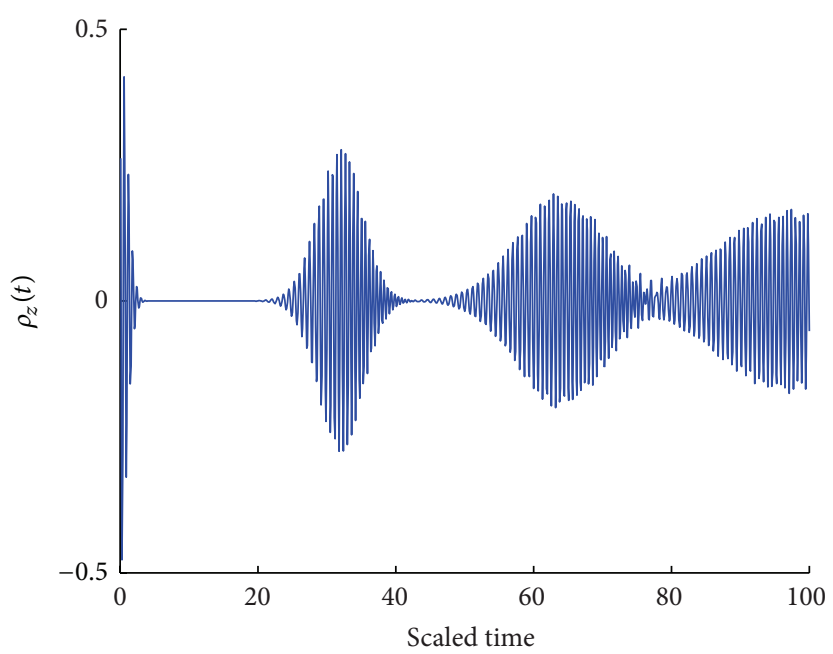

(a)

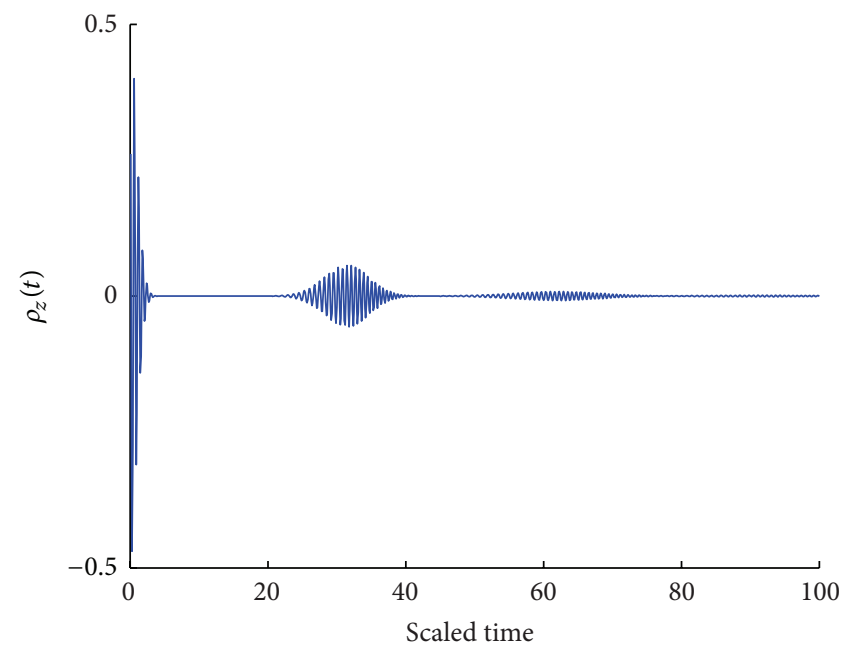

(c)

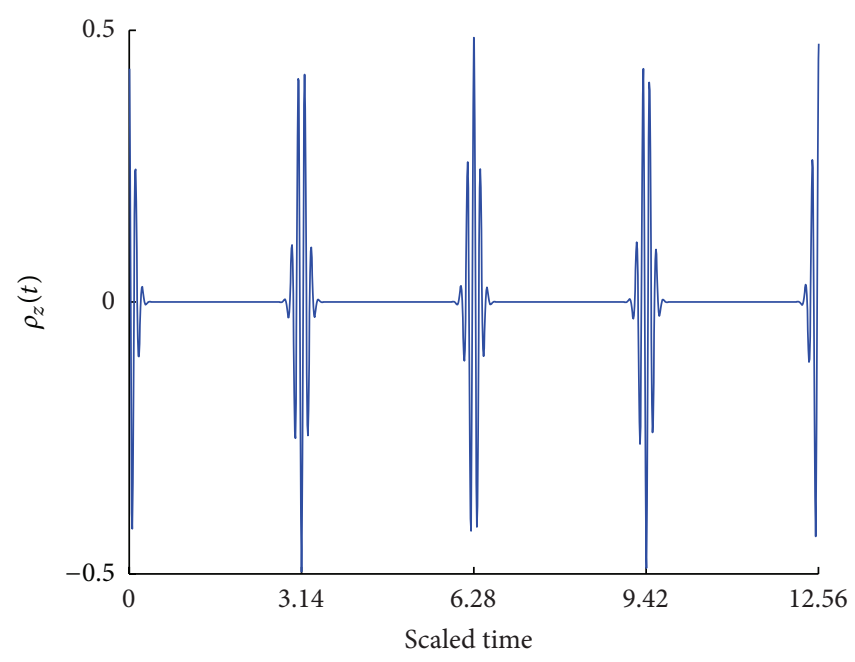

(b)

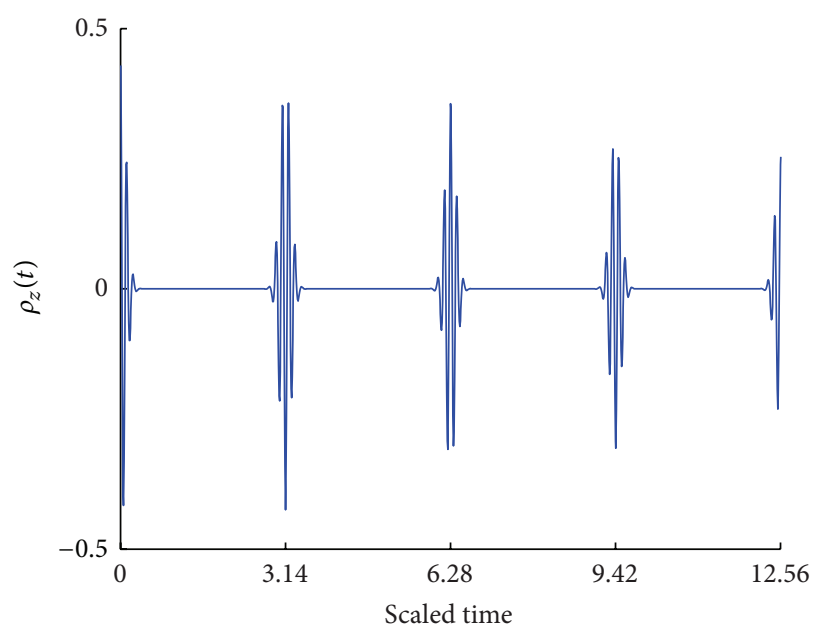

(d)

FIgURE 1: The evolution of the atomic population inversion $\rho_{z}(t)$ as a function of the dimensionless time $\lambda t$ for $\alpha=5$ and with different values of the number of photon transition $k$ and atomic damping parameter $\gamma / \lambda$ where $(\mathrm{a})(k, \gamma / \lambda)=(1,0.0001),(\mathrm{b})(k, \gamma / \lambda)=(1,0.1)$, (c) $(k, \gamma / \lambda)=(2,0.0001)$, and $(\mathrm{d})(k, \gamma / \lambda)=(2,0.1)$.

One can easily check that the $Q_{A}$ is normalized. The marginal atomic $Q$-function can be obtained by integrating the atomic $Q$-function $Q_{A}$ over the atomic variable $\Theta$ as follows:

$$
Q_{\Phi}=\int_{0}^{2 \pi} Q_{A} \sin \Theta d \Theta .
$$

\section{Numerical Results}

In this section, we depict the atomic inversion, scaled atomic Wehrl entropy, and marginal atomic Q-function dynamics of a single two-level atom interacting with one-mode cavity field under the action of atomic damping for various for the case of one and two photon process. One wants to discover the relation between the dynamics of the quantum entanglement quantified by the scaled atomic Wehrl entropy
(16) and marginal atomic Q-function (18), in particular, and understand the changes in behavior in the dynamics of different kinds of quantifiers and atomic inversion (13). In all of our plots, we take the average photon number $\bar{n}=|\alpha|^{2}=25$ and we recall that the time $t$ has been dimensionless; one of the time units is given by the inverse of the coupling constant $\lambda$.

In Figure 1, we plot the dynamics of the atomic population inversion $\hat{\rho}_{z}(t)$ as a function of the dimensionless time $\lambda t$ for different values of the atomic damping parameter $\gamma / \lambda$ and the number of photon transition $k$. In Figure 1(a), we assume that $k=1$ and consider the case of absence of the effect of the atomic damping parameter, that is, $\gamma / \lambda=0$. The collapse and revival phenomena is observed during the time evolution, where the revival appears at $\lambda t=2 m \pi \alpha$, where $m=0,1,2, \ldots$ On the other hand, we have a long collapse period between any two revivals, which are being short as 


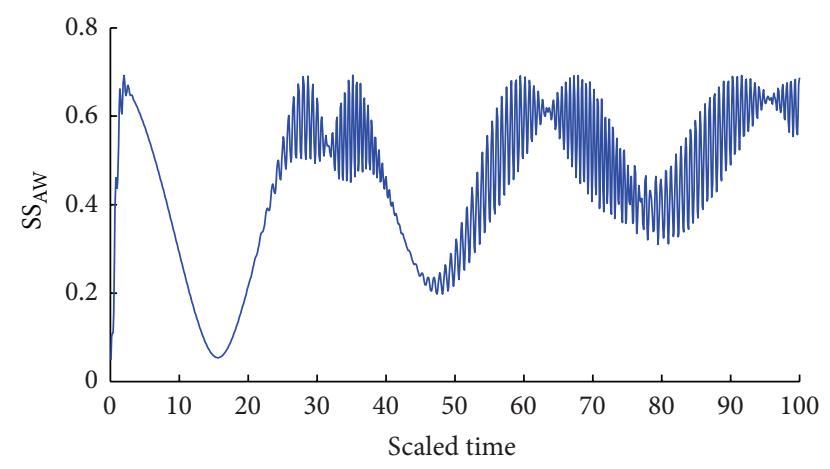

(a)

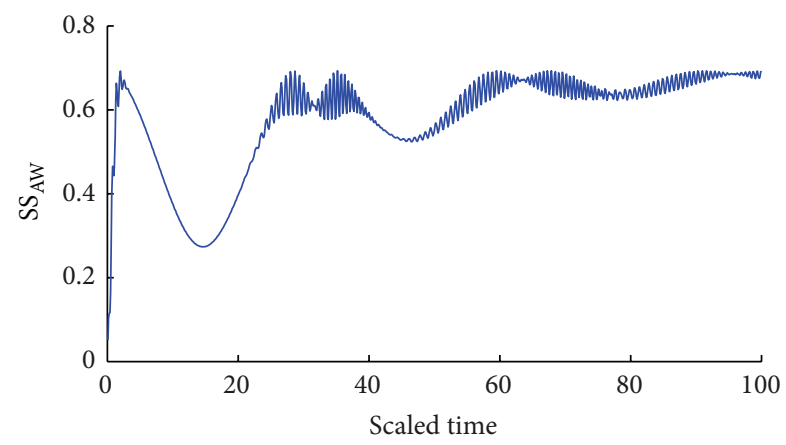

(b)

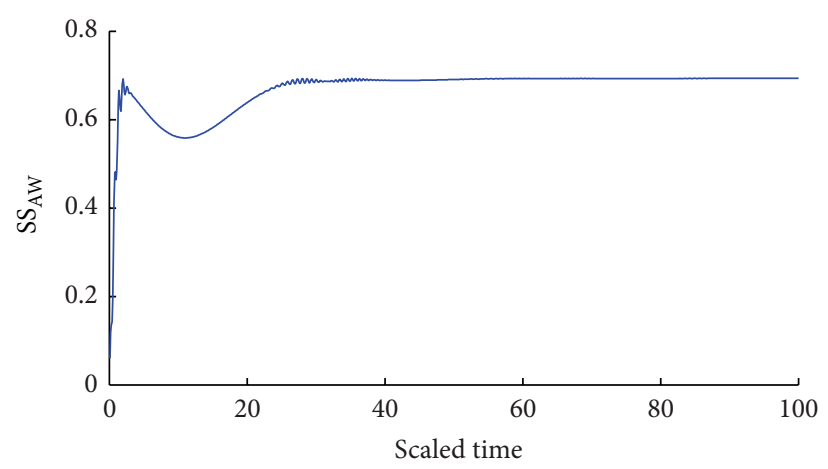

(c)

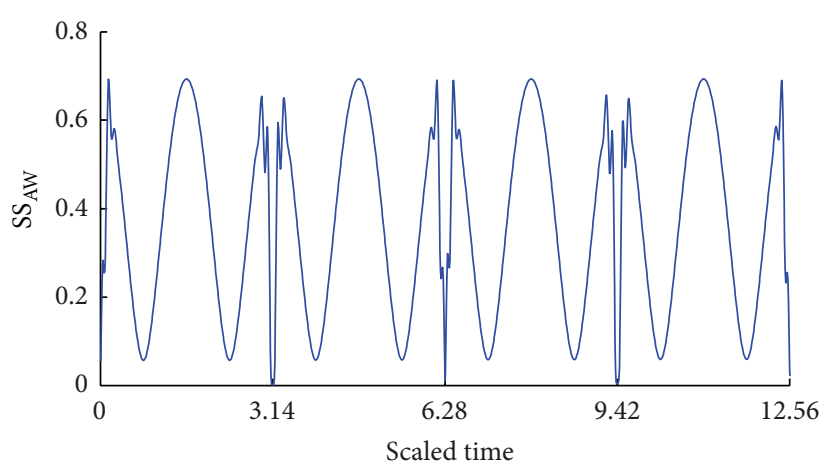

(d)

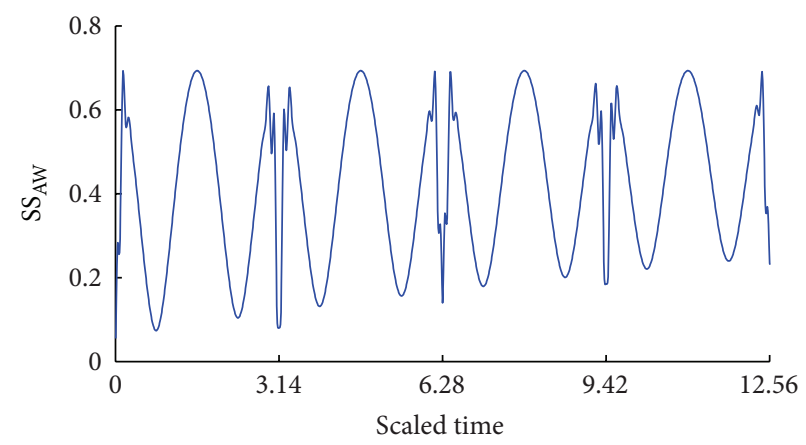

(e)

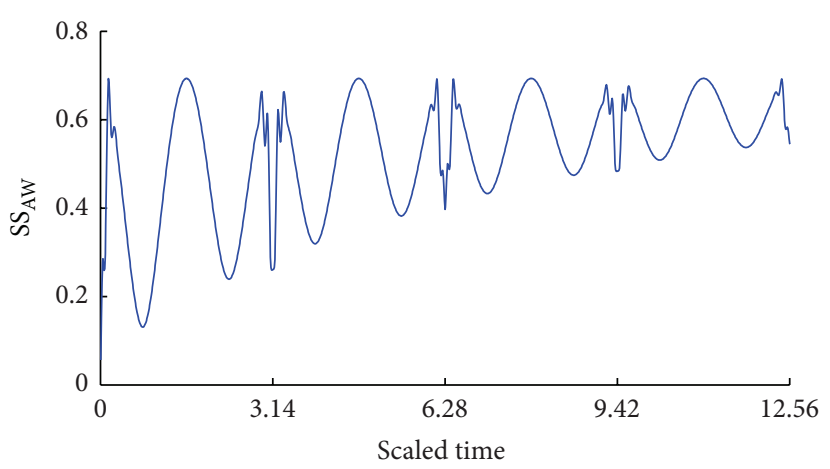

(f)

FIGURE 2: The evolution of the scaled atomic Wehrl entropy $\mathrm{SS}_{\mathrm{AW}}(t)$ as a function of the dimensionless time $\lambda t$ for $\alpha=5$ and with different values of the atomic damping parameter $\gamma / \lambda$ and number of photon transition $k$ where ((a), (b), (c)) $\gamma / \lambda=(0.0001,0.05,0.1)$ and ((d), (e), (f)) are the same as ((a), (b), (c)) but $k=2$.

the dimensionless time goes on. Figure 1(c) depicts the effect of $\gamma / \lambda$ on the dynamics of the atomic population inversion $\hat{\rho}_{z}(t)$. Also, the amplitude of the $\hat{\rho}_{z}(t)$ oscillations decreases and $\hat{\rho}_{z}(t)=0$, that is, $\left\langle e\left|\hat{\rho}_{A}(t)\right| e\right\rangle=\left\langle g\left|\hat{\rho}_{A}(t)\right| g\right\rangle$, when the dimensionless time is significantly large. In order to examine the effect of increasing the number of photon transition, we set $k=2$ in Figures 2(b) and 2(d). It is noticed that $\hat{\rho}_{z}(t)$ has a periodic and regular behavior where the periodic time is $2 \pi$. In this case, the effect of atomic damping parameter leads to decreasing the amplitude of $\hat{\rho}_{z}(t)$ oscillations gradually during the time evolution (see Figure 1(d)).

Now, it is the time to discuss the effect of the number of photons transition and atomic damping on the dynamical properties of the $\mathrm{SS}_{\mathrm{AW}}$. Figures 2(a), 2(b), and 2(c) represent the $\mathrm{SS}_{\mathrm{AW}}$ as a function of the scaled time $\lambda t$ when the atom interacts with one photon from the field, that is, $k=1$, for three values of $\gamma / \lambda=(0,0.05,0.1)$. It is clear that the $\mathrm{SS}_{\mathrm{AW}}$ is sensitive to the atomic damping parameter $\gamma / \lambda$, where the amplitude of the $\mathrm{SS}_{\mathrm{AW}}$ decreased when $\gamma / \lambda=0.05$. Also, the long lived entanglement is observed when $\gamma / \lambda=$ 0.1 . Based on the above discussion, the system entanglement could be controlled by using the atomic damping effect. Figures 2(d), 2(e), and 2(f) depict the evolution of the $\mathrm{SS}_{\mathrm{AW}}$ when $k=2$ in the case of taking the atomic damping effect into consideration or not. In this case, the $\mathrm{SS}_{\mathrm{AW}}$ exhibit a periodic oscillatory behavior with the periodic time $\lambda t=$ $m \pi$, this means that the system returns to the separable state $\left(\mathrm{SS}_{\mathrm{AW}}=0\right)$ (see Figure 2(a)). It is clear that the $\mathrm{SS}_{\mathrm{AW}}$ is more 


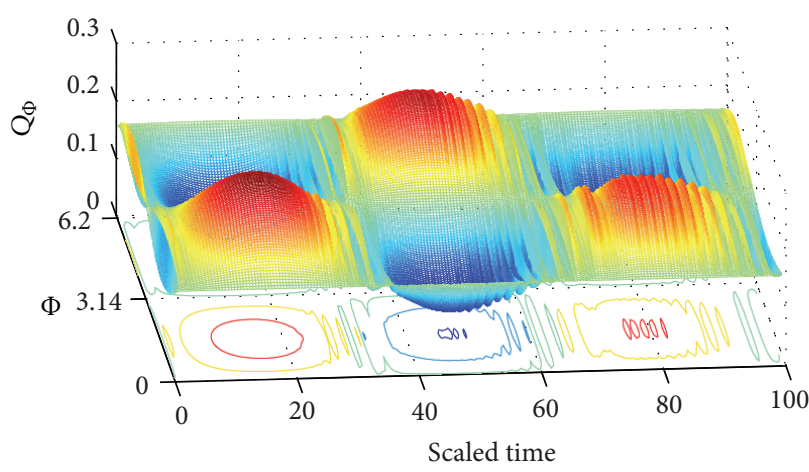

(a)

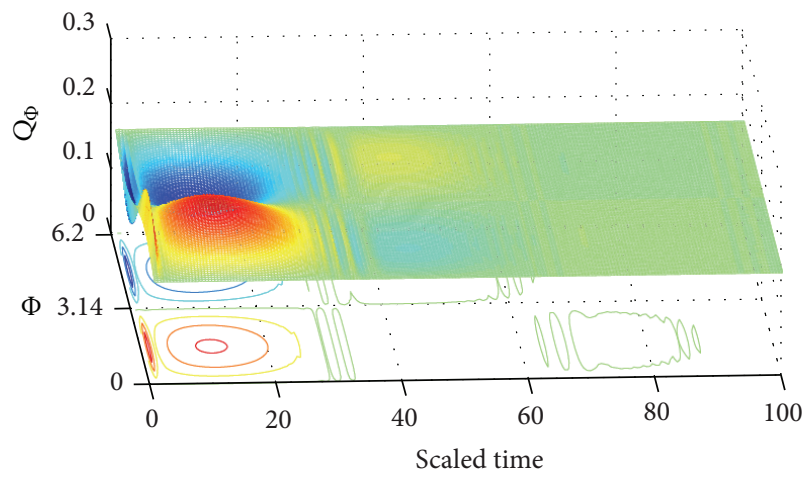

(c)

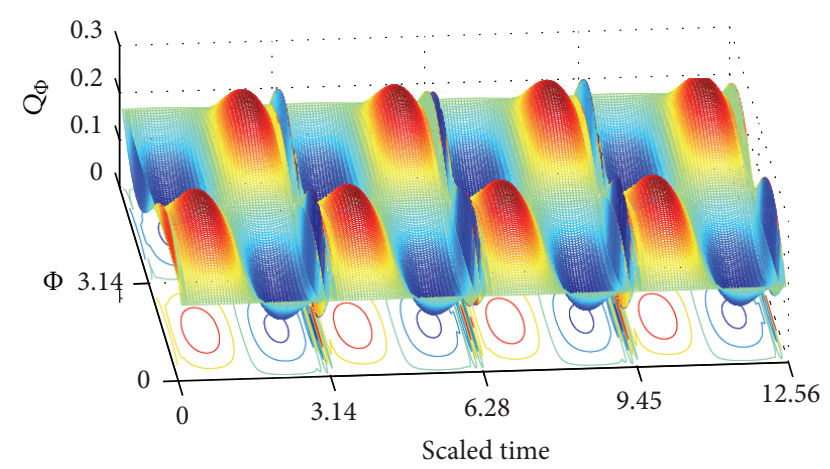

(b)

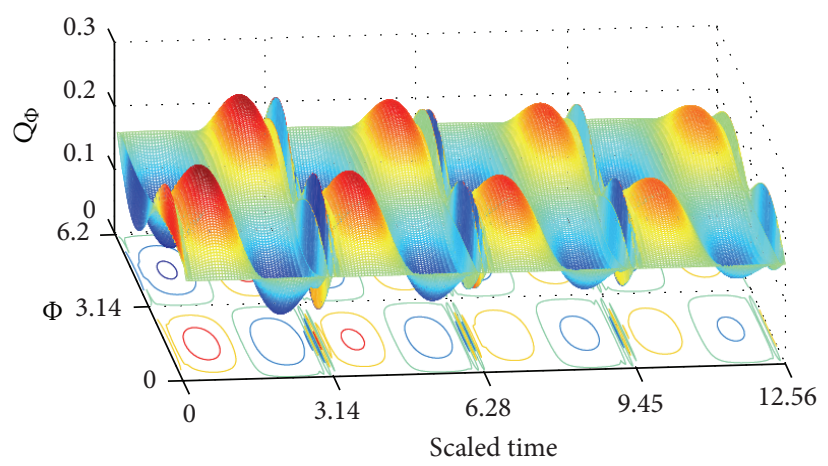

(d)

FIGURE 3: The surface plot of the marginal atomic $Q$-function $Q_{\Phi}(t)$ versus the dimensionless time $\lambda t$ and the atomic phase space parameter $\Phi$ for $\alpha=5$ and with different values of the number of photon transition $k$ and atomic damping parameter $\gamma / \lambda$ where $(\mathrm{a})(k, \gamma / \lambda)=(1,0.0001)$, (b) $(k, \gamma / \lambda)=(1,0.1),(\mathrm{c})(k, \gamma / \lambda)=(2,0.0001)$, and $(\mathrm{d})(k, \gamma / \lambda)=(2,0.1)$.

sensitive to the atomic damping $\gamma / \lambda$ when $k=1$ than when $k=2$ as shown in Figures 2(a), 2(c), 2(e), and 2(f).

In Figure 3, we consider the evolution of the marginal atomic $Q$-function $Q_{\Phi}(t)$ as a function of the dimensionless time $\lambda t$ and the atomic phase space parameter $\Phi$ for different values of the number of photon transition $k$ and atomic damping parameter $\gamma / \lambda$. It is observed that $Q_{\Phi}(t)$ oscillates between minimum and maximum values, where the local maximum peaks appear at $(\lambda t, \Phi)=((4 m+1)(\pi / 4), \pi / 2)$ or $((4 m+3)(\pi / 4), 3 \pi / 2)$, but the minimum peaks appear at $(\lambda t, \Phi)=((4 m+3)(\pi / 4), \pi / 2)$ or $((4 m+1)(\pi / 4), 3 \pi / 2)$. In other words, the exchange of the maximum and minimum values of $Q_{\Phi}$ is due to the variation of $\Phi$ which can be interpreted as follows. If $\Phi=\pi / 2$, we see that $Q_{\Phi}=X+Y$, while $Q_{\Phi}=X-Y$ if $\Phi=3 \pi / 2$. This also refers to the oneto-one correspondence between the behavior of $Q_{\Phi}(t)$ and the general behavior of the entanglement quantified by $\mathrm{SS}_{\mathrm{AW}}$ of the system under consideration see. This new observation suggests new ideas for using $Q_{\Phi}(t)$ as an entanglement indicator.

\section{Conclusion}

Quantum information technology largely relies on a precious and fragile resource, called quantum entanglement, which exhibits a highly nontrivial manifestation of the coherent superposition of states of composite quantum systems. However, our knowledge of the time evolution of this resource under realistic conditions, that is, when corrupted by environment-induced damping, is so far limited, and general statements on entanglement dynamics in open systems are scarce. In the present work, we will describe quantitatively the quantum entanglement between an atom and coherent field in terms of multiphoton and atomic damping processes especially, considering a two-level atom interaction with a single-mode quantized field in a coherent state and taking into account the number of multiphoton transition and atomic damping effect.

In this paper, we have studied the atomic inversion, scaled atomic Wehrl entropy, and marginal atomic Q-function dynamics of more realistic model including two-level atom interacting with a single mode cavity field in the presence and absence of the atomic damping effect for the case of one- and two-photon transition. As a quantifier, we have used the scaled atomic Wehrl entropy and marginal atomic Qfunction and investigated their behavior in terms of different parameters involved in the atom-field state.

We consider two distinct cases of the dynamics of $\mathrm{SS}_{\mathrm{AW}}$ and $Q_{\Phi}$ for field-atom interaction, when the atomic damping effect is neglected and considered. For both cases, we find that both of $S_{A W}$ and $Q_{\Phi}$ are good quantifiers of entanglement 
of the system exhibiting a monotonic behavior during the time evolution. In the absence of atomic damping parameter and the case of one-photon processes, the entanglement quantified by the $\mathrm{SS}_{\mathrm{AW}}$ and $\mathrm{Q}_{\Phi}$ oscillates between a maximum and minimum values which indicants the atom-field state. In the case of two-photon processes, we have found that $\mathrm{SS}_{\mathrm{AW}}$ and $Q_{\Phi}$ involve a regular and periodic behavior and the system returns to the sparable state, that is, $\mathrm{SS}_{\mathrm{AW}}=$ 0 , at the periodic dimensionless time. On the other hand, the entanglement quantified by $\mathrm{SS}_{\mathrm{AW}}$ is very sensitive to the atomic damping effect and the behavior is completely changed, where there is long lived entanglement between the atom and field. Moreover, $Q_{\Phi}$ is very sensitive to atomic damping parameter and may have a monotonic correlation between $S_{\mathrm{AW}}$ and $Q_{\Phi}$.

It is well known that the study of the physical properties of the atom-field interaction is a central and important problem in quantum optics and information. In this regard, our results show that the field-atom interaction considering the multiphoton processes and atomic damping effect have much richer structure. An important future investigation will be the study of the effect of the phase and cavity damping on the evolution of scaled atomic Wehrl entropy and marginal atomic Q-function.

\section{References}

[1] M. A. Nielsen and I. L. Chuang, Quantum Computation and Quantum Information, Cambridge University Press, Cambridge, UK, 2000.

[2] C. H. Bennett, G. Brassard, C. Crépeau, R. Jozsa, A. Peres, and W. K. Wootters, "Teleporting an unknown quantum state via dual classical and Einstein-Podolsky-Rosen channels," Physical Review Letters, vol. 70, no. 13, pp. 1895-1899, 1993.

[3] P. Agrawal and A. Pati, "Perfect teleportation and superdense coding with $W$ states," Physical Review A, vol. 74, no. 6, Article ID 062320, 5 pages, 2006.

[4] Z.-Q. Yin, H.-W. Li, W. Chen, Z.-F. Han, and G.-C. Guo, "Security of counterfactual quantum cryptography," Physical Review A, vol. 82, no. 4, Article ID 042335, 6 pages, 2010.

[5] Z.-R. Lin, G.-P. Guo, T. Tu, F.-Y. Zhu, and G.-C. Guo, "Counterfactual quantum cryptography," Physical Review Letters, vol. 103, no. 23, Article ID 230501, 4 pages, 2009.

[6] T. Morimae, "Strong entanglement causes low gate fidelity in inaccurate one-way quantum computation," Physical Review A, vol. 81, no. 6, Article ID 060307, 4 pages, 2010.

[7] K. Berrada, S. Abdel-Khalek, and C. H. Raymond Ooi, "Quantum metrology with entangled spin-coherent states of two modes," Physical Review A, vol. 86, no. 3, Article ID 033823, 5 pages, 2012.

[8] H. M. Wisemann and G. J. Milburn, Quantum Measurement and Control, Cambridge University Press, Cambridge, UK, 2010.

[9] E. T. Jaynes and F. W. Cummings, "Comparison of quantum and semiclassical radiation theories with application to the beam maser," Proceedings of the IEEE, vol. 51, no. 1, pp. 89-109, 1963.

[10] S. M. Barnett and P. L. Knight, "Dissipation in a fundamental model of quantum optical resonance," Physical Review A, vol. 33, no. 4, pp. 2444-2448, 1986.
[11] R. R. Puri and G. S. Agarwal, "Finite-Q cavity electrodynamics: dynamical and statistical aspects," Physical Review A, vol. 35, no. 8, pp. 3433-3449, 1987.

[12] T. Quang, P. L. Knight, and V. Buzek, "Quantum collapses and revivals in an optical cavity," Physical Review A, vol. 44, no. 9, pp. 6092-6096, 1991.

[13] J. Eiselt and H. Risken, "Quasiprobability distributions for the Jaynes-Cummings model with cavity damping," Physical Review A, vol. 43, no. 1, pp. 346-360, 1991.

[14] B.-G. Englert, M. Naraschewski, and A. Schenzle, "Quantumoptical master equations: an interaction picture," Physical Review A, vol. 50, no. 3, pp. 2667-2679, 1994.

[15] J. von Neumann, Mathematical Foundations of Quantum Mechanics, Princeton University Press, Princeton, NJ, USA, 1955.

[16] C. E. Shannon and W. Weaver, Mathematical Theory of Communication, Urbana University Press, Chicago, Ill, USA, 1949.

[17] A.-S. Obada and S. Abdel-Khalek, "New features of the atomic Wehrl entropy and its density in multi-quanta two-level system," Journal of Physics A, vol. 37, no. 25, article 6573, 2004.

[18] S. Abdel-Khalek, "Atomic wehrl entropy in a two-level atom interacting with a cavity field," Applied Mathematics and Information Sciences, vol. 1, no. 1, pp. 53-64, 2007.

[19] R. Dermez and S. Abdel-Khalek, "Atomic Wehrl entropy and negativity as entanglement measures for qudit pure states in a trapped ion," Journal of Russian Laser Research, vol. 32, no. 3, pp. 287-297, 2011.

[20] S. Abdel-Khalek, E. M. Khalil, and S. I. Ali, "Entanglement of a two-level atom papered in a finite trio-coherent state," Laser Physics, vol. 18, no. 2, pp. 135-143, 2008.

[21] S. Abdel-Khalek, H. F. Abdel-Hameed, and M. Abdel-Aty, "Atomic wehrl entropy of a single qubit system," International Journal of Quantum Information, vol. 9, no. 3, pp. 967-979, 2011.

[22] S. Abdel-Khalek, "Dynamics of fisher information in kerr medium," International Journal of Quantum Information, vol. 7, no. 8, pp. 1541-1548, 2009.

[23] A.-S. F. Obada, S. Abdel-Khalek, and A. Plastino, "Information quantifiers' description of weak field vs. strong field dynamics for a trapped ion in a laser field," Physica A, vol. 390, no. 3, pp. 525-533, 2011.

[24] A.-S. F. Obada and S. Abdel-Khalek, "Entanglement evaluation with atomic Fisher information," Physica A, vol. 389, no. 4, pp. 891-898, 2010.

[25] P. Goy, J. M. Raimond, M. Gross, and S. Haroche, "Observation of cavity-enhanced single-atom spontaneous emission," Physical Review Letters, vol. 50, no. 24, pp. 1903-1906, 1983.

[26] D. Meschede, H. Walther, and G. Müller, "One-atom maser," Physical Review Letters, vol. 54, no. 6, pp. 551-554, 1985.

[27] B. W. Shore and P. L. Knight, "The Jaynes-Cummings model," Journal of Modern Optics, vol. 40, no. 7, pp. 1195-1238, 1993.

[28] I. Marzoli, J. I. Cirac, R. Blatt, and P. Zoller, "Laser cooling of trapped three-level ions: designing two-level systems for sideband cooling," Physical Review A, vol. 49, no. 4, pp. 27712779, 1994.

[29] H. A. Hessian and A.-B. A. Mohamed, "Entanglement and its dynamics with atomic spontaneous decay," Chinese Physics Letters, vol. 25, no. 7, article 2492, 2008.

[30] S. Abdel-Khalek and A.-S. F. Obada, "The atomic Wehrl entropy of a V-type three-level atom interacting with two-mode squeezed vacuum state," Journal of Russian Laser Research, vol. 30, no. 2, pp. 146-156, 2009. 

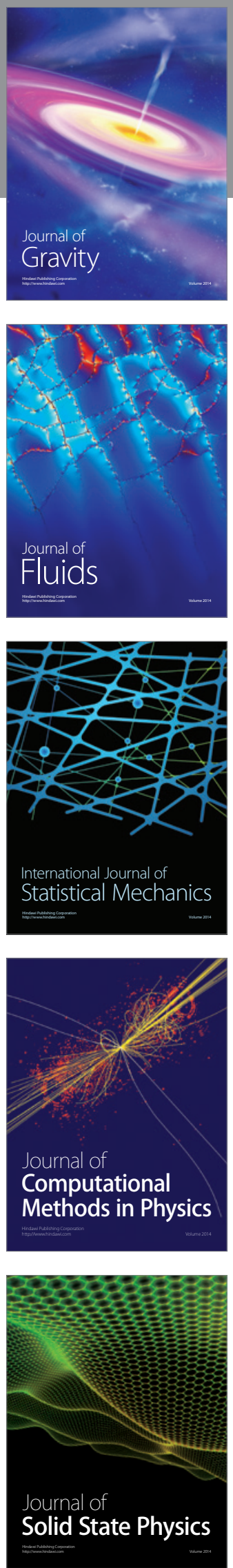

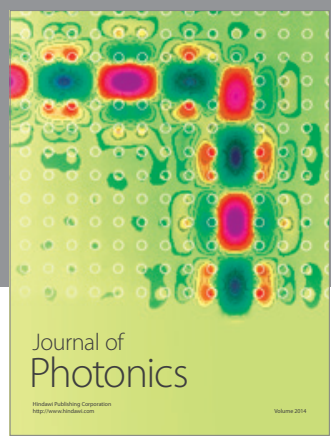

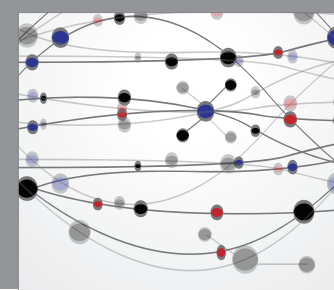

The Scientific World Journal

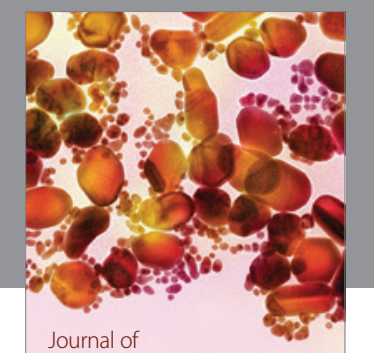

Soft Matter
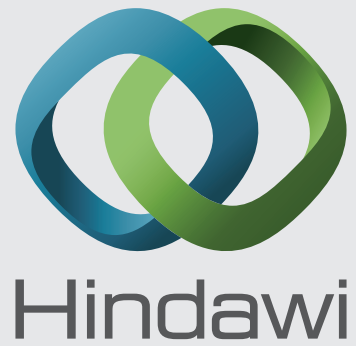

Submit your manuscripts at

http://www.hindawi.com
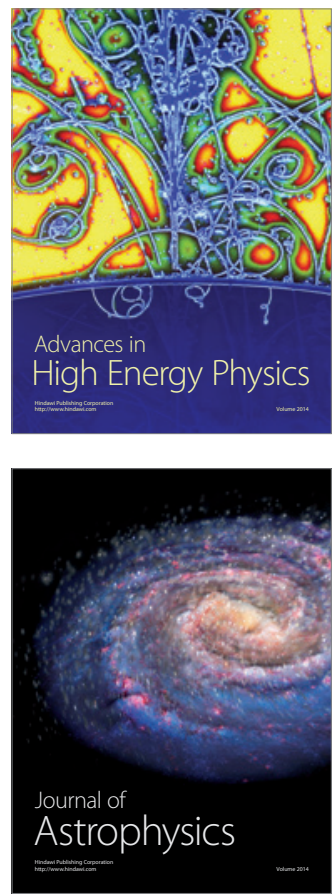
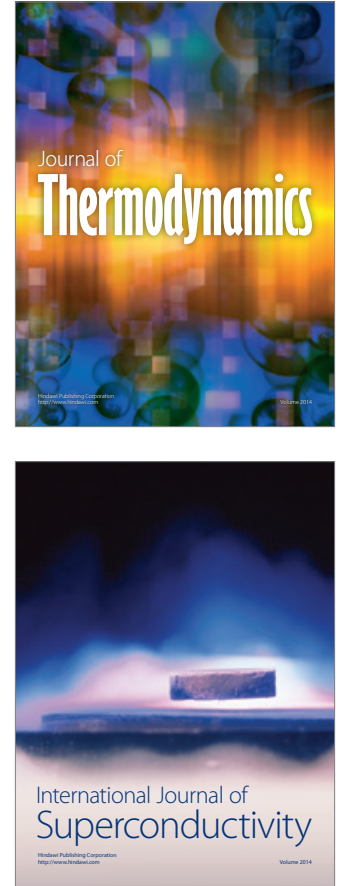
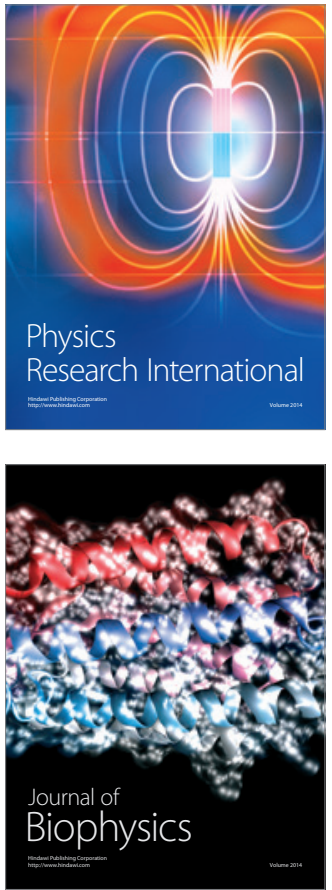
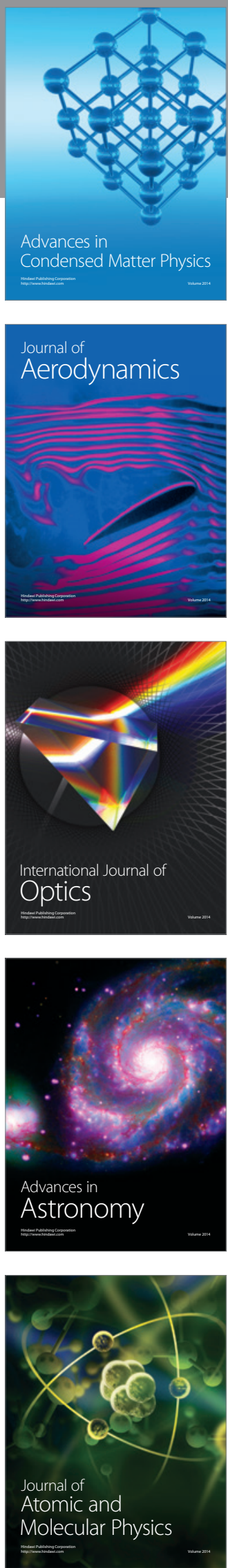